\title{
Desigualdades socioeconómicas en la mortalidad por enfermedades cardiovasculares: Región Pacifico de Colombia, 2002-2015
}

\author{
Socioeconomic inequalities in mortality due to cardiovascular \\ diseases: Pacific Region of Colombia, 2002-2015
}

Mauricio Pérez-Flórez (https://orcid.org/0000-0003-0219-9773) ${ }^{1}$

Jorge Alberto Achcar (https://orcid.org/0000-0002-9868-9453) ${ }^{2}$

${ }^{1}$ Grupo de Investigación en Economía, Gestión y Salud (ECGESA), Departamento de Salud Pública y Epidemiología, Facultad de Ciencias de la Salud, Pontificia Universidad Javeriana, Cali. Calle 18 No.118 - 250 Cali, Tercer Piso, Edificio Raúl Posada. Cali Valle del Cauca Colombia.mauricioperez@ javerianacali.edu.co

${ }^{2}$ Faculdade de Medicina de Ribeirão Preto, Universidade de São Paulo. Ribeirão Preto SP Brasil.

\begin{abstract}
Cardiovascular diseases (CVD) are the leading cause of death in the world, and they are considered a serious public health problem in Colombia. The main goal of this study was to analyze CVD mortality spatially and temporarily in the Pacific region of Colombia during the 2002-2015 period, and its association with some municipal socio-economic indicators using spatial statistical analysis techniques. It involved a descriptive-ecological study in the 177 municipalities of the $\mathrm{Pa}$ cific region that used CVD mortality data, under codes I00-I99 of the International Classification of Diseases (ICD-10), and seven municipal socio-economic indicators. The analysis included the calculation of crude and standardized mortality rates, according to sex, for CVD and its main causes, and modeling of CVD death counts using Bayesian hierarchical models. During the 20022015 period, standardized rates of CVD mortality showed a downward trend in men (129.0 to 119.3 ) and in women (129.0 to 110.0), the main causes of death being ischemic heart diseases, followed by cerebrovascular diseases. In general, the risk of CVD mortality was higher in the less economically and socially privileged municipalities. Key words Mortality, Cardiovascular Diseases, Health Status Disparities, Spatial Analysis, Colombia
\end{abstract}

Resumen Las enfermedades cardiovasculares (ECV) son la principal causa de muerte en el mundo y en Colombia siendo consideradas un serio problema de salud pública. El objetivo de este estudio fue analizar espacial y temporalmente la mortalidad por ECV en la región Pacifico de Colombia durante el periodo 2002-2015 y su asociación con algunos indicadores socioeconómicos municipales usando técnicas estadísticas de análisis espacial. Fue un estudio descriptivo-ecológico en los 177 municipios de la región Pacifico que usó datos de mortalidad por ECV, bajo los códigos I00-I99 de la Clasificación Internacional de Enfermedades (CIE-10), y siete indicadores socioeconómicos municipales. El análisis incluyó cálculo de tasas de mortalidad crudas y estandarizadas, según sexo, para las ECV y sus principales causas, y la modelación del conteo de defunciones usando modelos jerárquicos Bayesianos. Durante el periodo 2002-2015 las tasas estandarizadas de mortalidad por ECV mostraron una tendencia descendente en hombres (129,0 a 119,3) y en mujeres (129,0 a 110,0) siendo las principales causas de muerte las enfermedades isquémicas del corazón, seguidas de las cerebrovasculares. En general, el riesgo de mortalidad por ECV fue mayor en los municipios menos favorecidos económica y socialmente.

Palabras clave Mortalidad, Enfermedades Cardiovasculares, Desigualdades en la Salud, Análisis Espacial, Colombia 


\section{Introducción}

Las enfermedades cardiovasculares (ECV) o enfermedades del sistema circulatorio son la principal causa de muerte en el mundo y en Colombia, siendo consideradas un serio problema de salud pública. Las ECV junto con el cáncer, diabetes y enfermedades respiratorias crónicas conforman el grupo de enfermedades no transmisibles (ENT) que comparten factores de riesgo comportamentales (consumo excesivo de alcohol, sedentarismo, ingesta de sal/sodio y uso de tabaco) y metabólicos (presión arterial y glucosa en sangre elevadas, y obesidad $)^{1}$, soportan la mayor carga de mortalidad mundial y traen consecuencias negativas en términos humanos, sociales y económicos ${ }^{2}$. En 2016 las ENT fueron responsables del 71\% (41/57 millones) de las muertes mundiales donde las ECV lideraron las causas de muerte con 17,9 millones de defunciones que representaron el $31 \%$ de las muertes y el $44 \%$ de las ENT ${ }^{1}$. Para el año 2015 las tasas estandarizadas de mortalidad por ECV (x 100.000) fueron estimadas en 286 a nivel mundial y en 157 para América Latina Andina ${ }^{3}$. Las ENT dominan la mortalidad en los países desarrollados, mientras que otros en desarrollo donde se incluyen algunos países de América Latina soportan una alta carga de enfermedades infecciosas en presencia de un aumento de ENT ${ }^{4}$.

En Colombia desde la década de los 1980's las ECV han liderado las principales causas de muerte, siendo la más importante las enfermedades isquémicas cardiacas, además de otras ENT ubicadas entre las diez primeras como el accidente cerebrovascular, la diabetes y la enfermedad hipertensiva $^{5}$. Durante el periodo 1998-2011 la tasa estandarizada de mortalidad por ECV fue de 104,6 defunciones x 100.000 con una clara tendencia descendente de 108,6 defunciones en 1998 a 95,9 en 2011. Sin embargo, su importancia relativa como causa de muerte aumentó del $21,6 \%$ al $25,4 \%$ durante ese periodo ${ }^{6}$, además de observar diferencias en la mortalidad por ECV entre sexo y regiones de Colombia. Durante el periodo 19982011 se observó mayor riesgo de mortalidad por ECV en hombres, además que el departamento del Valle del Cauca presentó las mayores tasas de mortalidad de ECV del país?

La distribución no uniforme de diversos indicadores de salud (morbilidad y mortalidad) entre grupos poblacionales es el tema principal de las desigualdades o inequidades en salud. En consenso, las inequidades en salud se definen como diferencias en el estado de salud entre gru- pos poblacionales definidos por características demográficas, sociales, económicas o geográficas, y que además se consideran innecesarias, injustas y evitables ${ }^{8}$. Uno de los determinantes más importantes de la salud es el espacio geográfico donde habitan las personas que, de acuerdo con sus características físicas, sociales o económicas, afectan diferencialmente la salud poblacional ${ }^{9}$.

En Colombia el estudio de las desigualdades sociales en la mortalidad según áreas geográficas ha tratado temas como las causas evitables ${ }^{10}$, cáncer de mama y cuello uterino ${ }^{11,12}$, VIH y tumores malignos $^{13}$, mortalidad infantil ${ }^{14-16} \mathrm{y}$ materna ${ }^{17,18}$, causas externas ${ }^{9}$, enfermedades cardiovasculares $^{19}$ o mortalidad por diversas causas ${ }^{20,21}$. Estos estudios se han caracterizado por sus diseños ecológicos con diferentes unidades de agregación: departamento, subregión, municipio o localidad. No obstante, en la gran mayoría de ellos su nivel de análisis se queda en lo descriptivo siendo muy pocas las investigaciones que han introducido las técnicas de análisis espacial en el estudio de las desigualdades geográficas en salud en Colombia.

La región Pacifico de Colombia, conformada por cuatro departamentos (Chocó, Valle del Cauca, Cauca y Nariño) (Figura 1), se caracteriza por sus contrastes sociales y económicos donde el Valle del Cauca es el tercer departamento más desarrollado e industrializado de Colombia, mientras el Chocó y Cauca están dentro de aquellos que presentan los peores índices sociales, económicos y de salud del país ${ }^{22,23}$.

Considerando que las desigualdades en la mortalidad también ocurren en las ECV, al interior de los países y en regiones donde abunda la pobreza $^{24}$, el objetivo de este estudio fue analizar espacial y temporalmente la mortalidad por ECV en la región Pacifico de Colombia durante el periodo 2002-2015 y su asociación con indicadores socioeconómicos municipales usando técnicas de análisis espacial.

\section{Métodos}

\section{Tipo de estudio y área de estudio}

Fue un estudio con un componente descriptivo y otro ecológico longitudinal con municipio como unidad de análisis (177 en total) en los cuatro departamentos que conforman la región $\mathrm{Pa}$ cifico de Colombia: Chocó, Valle del Cauca, Cauca y Nariño (Figura 1). Esta región se ubica en el occidente colombiano con aproximadamente 8.237.174 habitantes en 2005, un poco más del $17 \%$ de la población total del país ${ }^{25}$. $\mathrm{Al}$ interior 
a)

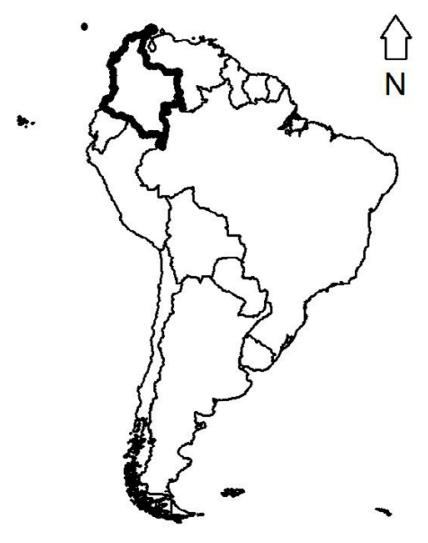

c)

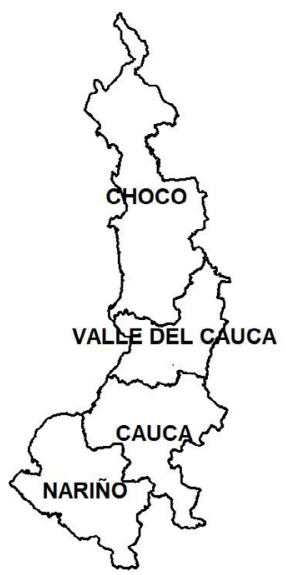

b)

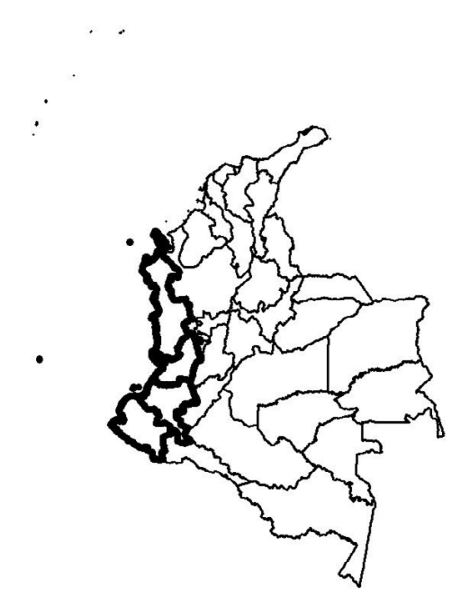

d)

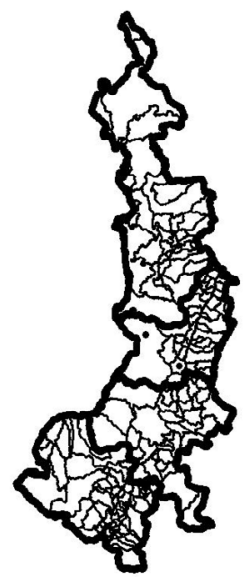

Figura 1. Área de estudio: a) Mapa de Suramérica mostrando a Colombia; b) Mapa de Colombia mostrando a los cuatro departamentos de la región Pacifico; c) Mapa de los cuatro departamentos; y d) Mapa mostrando los 177 municipios de la región Pacifico de Colombia.

Fuente: Desarrollada por los autores.

de Colombia esta región es reconocida por sus altos niveles de pobreza, desigualdades sociales y rezago de carácter industrial, económico y social $^{14,26}$. Por mencionar solo un ejemplo, en 2005 los departamentos con menores índices de Necesidades Básicas Insatisfechas (NBI) fueron Quindío $(11,8 \%)$, Valle del Cauca $(12,6 \%)$ y su capital Bogotá (6,6\%); mientras que Cauca $(23,3 \%)$ y Nariño $(28,7 \%)$ se ubicaron en el tercer cuartil; y Chocó presentó el peor NBI del país $(67,1 \%)$.

\section{Población de estudio y fuentes de información}

La población de estudio correspondió a defunciones no fetales de hombres y mujeres resi- dentes en el área de estudio durante el periodo 2002-2015. Se usaron fuentes secundarias donde los datos de mortalidad (registros individuales) se obtuvieron de las Estadísticas Vitales del Departamento Administrativo Nacional de Estadística (DANE) donde se consolida la información de certificados de defunción del país ${ }^{27}$.

Se incluyeron todas las defunciones por enfermedades del sistema circulatorio con base en la causa básica de muerte según la CIE-10 (códigos: $\mathrm{I00}-\mathrm{I} 99)^{28}$ y con lugar de residencia en los municipios del área de estudio $(\mathrm{N}=141.250)$. Se excluyeron 516 registros $(0,37 \%)$ con información faltante en las variables sexo y edad, y se analizaron 140.734 defunciones. Para el componente "ecológico" se agruparon las defunciones anuales 
por municipio. El número de habitantes por municipio, año, sexo y edad también se obtuvo del DANE.

\section{Variables del estudio}

Las variables del estudio fueron las contenidas en el certificado de defunción de las cuales se consideraron sexo (masculino/femenino); grupo de edad $(<1,2-4$, luego grupos quinquenales desde 5-9 hasta 75-79 y 80 años y más); municipio de residencia; y causa básica de la defunción codificada bajo la CIE-10. En Colombia esta información es recolectada habitualmente por personal médico al momento de la defunción.

La variable respuesta del estudio fue la mortalidad anual municipal por ECV. Las variables independientes fueron los indicadores socioeconómicos del Cuadro 1 que se seleccionaron por tener relevancia en el estudio de las desigualdades en salud y por estar disponibles a nivel de municipio. Una descripción detallada de los indicadores socioeconómicos, su periodo y fuente se presenta en el Cuadro 1.

\section{Análisis de la información}

El análisis descriptivo incluyó el cálculo de tasas de mortalidad según sexo (crudas y estandarizadas) por ECV y grupos específicos que incluyeron: enfermedades isquémicas del corazón (códigos CIE-10: I20-I25); enfermedades cerebrovasculares (I60-I69); enfermedades hipertensivas (I10-I15); enfermedad cardiopulmonar, enfermedades de la circulación pulmonar y otras formas de enfermedad del corazón (I26-I45, I47-I49, I51); insuficiencia cardiaca (I50); y otras enfermedades del sistema circulatorio (I00-I09, I46, I70, I71-I99). Las tasas estandarizadas usaron el método directo con población estándar la de Colombia en 2005. Todas las tasas fueron ampliadas $\mathrm{x} 100.000$ habitantes.

Para la comparación de las tasas de mortalidad municipales se calcularon tasas de mortalidad estandarizadas por el método indirecto que entrega la razón de mortalidad estandarizada (REM) comparando las muertes observadas $(Y)$ con las muertes esperadas $(E)^{29}$. Las muertes $E$ se calcularon tomando los riesgos de la región Pacifico durante 2002-2015 y en grupos quinquenales. La REM se multiplicó por 100 para facilitar su interpretación $(R M E=Y / E \times 100)$.

Para determinar la relación entre la mortalidad por ECV y las condiciones socioeconómicas de los municipios de la región Pacifico durante el periodo 2002-2015 se estimaron los parámetros de un modelo espacio-temporal basado en Achcar et al. ${ }^{30}$. La variable respuesta $Y_{i j}$ fue el número de muertes observadas por ECV en el municipio $i(i=1,2, \ldots, n)(\mathrm{n}=177$ municipios) y en el año $j$ $(j=1,2, \ldots, T)(\mathrm{T}=14$ años, correspondiente a los años 2002 a 2015). El modelo estadístico asume que $Y_{i j}$ sigue una distribución de Poisson con parámetro $\lambda_{i j}$

$$
Y_{i j} \mid \lambda_{i j} \sim \operatorname{Poisson}\left(\lambda_{i j}\right) ; i=1, \ldots, n ; j=1, \ldots, T
$$

Considerando los indicadores socioeconómicos (Cuadro 1) como covariables $\left(X_{1}\right.$ a $\left.X_{7}\right)$ se asumió el siguiente modelo de regresión para la distribución Poisson presentada en (1):

$$
\begin{aligned}
& \log \left(\lambda_{i j}\right)=\log N_{i j}+\beta_{0 j}+\beta_{1 j}\left(X_{1 i}\right)+ \\
& \beta_{2 j}\left(X_{2 i}-\bar{X}_{2}\right)+\beta_{3 j}\left(X_{3 i}-\bar{X}_{3}\right)+\beta_{4 j}\left(X_{4 i} \bar{X}_{4}\right)+ \\
& \beta_{5 j}\left(X_{5 i} \bar{X}_{5}\right)+\beta_{6 j}\left(X_{6 i} \bar{X}_{6}\right)+\beta_{7 j}\left(X_{7 i} \bar{X}_{7}\right)+ \\
& b_{i}+W_{i j}
\end{aligned}
$$

Para $i=1, \ldots, 177$ y $j=1, \ldots, 14 ; N_{i j}$ es el número de habitantes en el municipio $i$ y año $j ; \bar{X}_{1}, l=2$, ..., $k(\mathrm{k}=7)$ denota el promedio de la covariable $X_{l}$. Los parámetros $\beta_{0 j}$ a $\beta_{7 j}$ son los coeficientes de regresión y $\beta_{1 j}$ a $\beta_{7 j}$ representan las medidas de efecto de los respectivos indicadores socioeconómicos $X_{1}$ a $X_{7}$. El componente $b_{i}$ es un efecto aleatorio asociado al $i$-ésimo municipio que captura la posible correlación espacial entre la mortalidad de los municipios vecinos. El componente $W_{i j}$ representa un efecto aleatorio para capturar la tendencia temporal de los 14 años. El modelo de la ecuación (2) fue ajustado en la población total, en hombres y en mujeres, encontrando resultados muy similares.

El ajuste del modelo fue realizado bajo un enfoque Bayesiano usando el programa OpenBUGS versión 3.2.3

\section{Detalles de la modelación estadística}

Se asumió que $b_{i}$ tiene una distribución a priori espacial normal con estructura Autorregresiva Condicional (CAR), es decir:

$b_{i} \mid\left\{b_{j}, i \neq j, j \in A_{(\mathrm{i})}^{*}\right\}, \sigma_{\mathrm{b}}^{2} \sim N\left(\bar{\eta}_{i}, \frac{\sigma_{\mathrm{b}}^{2}}{\mathrm{n}_{\mathrm{i}}}\right.$

Donde $\mathrm{A}_{(\mathrm{i})}^{*}$ denota el conjunto de municipios vecinos al municipio $i ; n_{i}$ denota el número de elementos en $A_{(i)}^{*}$ o número vecinos del municipio $\bar{i} \eta_{i}$ es la media de los efectos aleatorios $b_{i}$ de los municipios vecinos al municipio $i$; y $\sigma_{\mathrm{b}}{ }^{2}$ es un parámetro desconocido que representa la varianza de la distribución. La notación $N(a, b)$ 
Cuadro 1. Indicadores sociales y económicos para los municipios del área de estudio.

\begin{tabular}{|c|c|c|c|c|}
\hline Indicador $\left[X_{l}\right]$ & Descripción del Indicador & Unidad & Periodo & Fuente \\
\hline $\begin{array}{l}\text { Categorización de } \\
\text { los municipios } \\
{\left[X_{1}\right]}\end{array}$ & $\begin{array}{l}\text { Es una clasificación de los municipios de acuerdo con } \\
\text { su número de habitantes e Ingresos Corrientes de Libre } \\
\text { Inversión (ICLD). Se codifican de } 0 \text { (grandes ciudades) a } \\
6 \text { (municipios menores). En este estudio se definió como } \\
\text { categoría de referencia (0) a los municipios de la categoría } \\
\text { "6" (161 municipios pequeños) y como exposición } \\
\text { (1) a los demás municipios de las categorías } 0 \text { a } 5 \text { ( } 16 \\
\text { municipios no pequeños) }\end{array}$ & 0 a 6 & $\begin{array}{l}1993-2012 \\
\text { (constante) }\end{array}$ & $\mathrm{DNP}$ \\
\hline $\begin{array}{l}\text { Índice de } \\
\text { Desarrollo } \\
\text { Humano (IDH }\left[X_{2}\right]\end{array}$ & $\begin{array}{l}\text { Este indicador mide el desarrollo de los municipios en } \\
\text { tres aspectos esenciales: esperanza de vida, nivel educativo } \\
\text { y PIB per cápita. Menores valores indica menor desarrollo }\end{array}$ & 0 a 1 & 2005 & PNUD \\
\hline $\begin{array}{l}\text { Necesidades } \\
\text { Básicas } \\
\text { Insatisfechas (NBI) } \\
{\left[X_{3}\right]}\end{array}$ & $\begin{array}{l}\text { Indica el porcentaje de la población que presenta al menos } \\
\text { una NBI de acuerdo con los indicadores seleccionados: } \\
\text { viviendas inadecuadas, viviendas con hacinamiento } \\
\text { crítico, viviendas con servicios inadecuados, viviendas } \\
\text { con alta dependencia económica, viviendas con niños en } \\
\text { edad escolar que no asisten a la escuela. Mayores valores } \\
\text { indican mayor pobreza }\end{array}$ & $\%$ & 2005 & DANE \\
\hline $\begin{array}{l}\text { Índice de Pobreza } \\
\text { Multidimensional } \\
(\mathrm{IPM}) \\
{\left[X_{4}\right]}\end{array}$ & $\begin{array}{l}\text { Mide el grado de privación de la población en un } \\
\text { conjunto de cinco dimensiones relacionadas con } \\
\text { condiciones educativas del hogar, condiciones de la niñez } \\
\text { y juventud, trabajo, salud y acceso a servicios públicos } \\
\text { domiciliarios y condiciones de la vivienda. Una persona } \\
\text { es multidimensionalmente pobre si no supera la tercera } \\
\text { parte de las dimensiones seleccionadas (5/15) o no supera } \\
\text { el } 33,3 \% \text { según la ponderación asignada. Mayores valores } \\
\text { indican mayor pobreza }\end{array}$ & $\%$ & 2005 & $\mathrm{DNP}$ \\
\hline $\begin{array}{l}\text { Déficit de Vivienda } \\
(\mathrm{DEVI}) \\
{\left[X_{5}\right]}\end{array}$ & $\begin{array}{l}\text { Mide los hogares que se encuentran en déficit de vivienda, } \\
\text { ya sea cuantitativo o cualitativo. Corresponde a la suma de } \\
\text { déficit de vivienda cuantitativo (\%) + déficit de vivienda } \\
\text { cualitativo (\%). Mayores valores indican mayor déficit de } \\
\text { vivienda } \\
\text { • El DEVI cuantitativo mide si la cantidad de viviendas } \\
\text { existentes es insuficiente para albergar a la totalidad de la } \\
\text { población del país } \\
\text { - El DEVI cualitativo mide si las viviendas existentes no } \\
\text { cumplen con estándares mínimos de calidad y por ende } \\
\text { no ofrecen una calidad de vida adecuada a sus moradores }\end{array}$ & $\%$ & 2005 & DANE \\
\hline $\begin{array}{l}\text { Cobertura en salud } \\
{\left[X_{6}\right]}\end{array}$ & $\begin{array}{l}\text { Mide el porcentaje de la población que posee } \\
\text { aseguramiento en salud. Mayores valores indican mayor } \\
\text { proporción de población asegurada }\end{array}$ & $\%$ & 2005 & DNP \\
\hline $\begin{array}{l}\text { Tasa de alfabetismo } \\
(\%) \\
{\left[X_{7}\right]}\end{array}$ & $\begin{array}{l}\text { Mide el porcentaje de la población de } 15 \text { años o más que } \\
\text { declaran saber leer y escribir, con base al censo } 2005 . \\
\text { Mayores valores indican mayor oportunidad de educación }\end{array}$ & $\%$ & 2005 & DANE \\
\hline
\end{tabular}

DPN: Departamento Nacional de Planeación; DANE: Departamento Administrativo Nacional de Estadística; PNUD: Programa de las Naciones Unidas para el Desarrollo, Colombia.

Fuente: Desarrollada por los autores.

representa una distribución normal con media $a$ y varianza $b$. El criterio usado para la definición de los vecinos fue el de adyacencia (matriz Queen de contigüidad).

En el modelo de regresión (2) $W_{i j}$ representa un efecto aleatorio para capturar la tendencia temporal, el cual es asumido como un proceso
Gaussiano con distribución normal multivariada (NM) dada por:

$$
W_{i j} \sim N M(\overrightarrow{0} ; \Sigma)
$$

Donde $\overrightarrow{0}$ es un vector de medias (vector de ceros) $14 x 1$ y $\Sigma$ es la matriz de varianza-covarianza $14 \times 14$ : 


$$
\Sigma=\left[\operatorname{Cov}\left(W_{i j}, W_{i j}^{*}\right)\right.
$$

Donde los elementos de (5) son:

$$
\operatorname{Cov}\left(W_{i j}, W_{i j}^{*}\right)=\theta_{1} \exp \left(-\sum_{k=2}^{K} \theta_{\mathrm{k}}\left|j-\mathrm{j}^{*}\right|^{\mathrm{k}-1}\right)
$$

Para $j=1, \ldots, 14, k=1,2, \ldots, K$. El valor de $K$ fue fijo $(K=2)$ después de asumir varios valores. El parámetro $\theta_{\mathrm{k}}$ es desconocido.

Se asumieron las siguientes distribuciones a priori para los parámetros del modelo (2):

- Para los parámetros $\beta_{l j}(l=0,1, \ldots, 7$ y $j=1$, ...,14) una distribución normal no informativa, es decir $\beta_{l j} \sim N\left(0 ; c_{l j}^{2}\right)$. Se asumió el hiperparámetro $c_{l j}=0,01$.

- Para $\sigma_{\mathrm{b}}^{2}$ se asumió una distribución Gamma con media $a / b$ y varianza $a / b^{2}$, es decir $\sigma_{\mathrm{b}}{ }^{2} \sim$ $\operatorname{Gamma}(a ; b)$. Se asumió $a=b=10$.

- Para $\theta_{\mathrm{k}}(k=1,2, \ldots, K)$ se asumió una distribución uniforme entre cero y $d_{k}$, es decir, $\theta_{\mathrm{k}} \sim \mathrm{U}(0$; $\mathrm{d}_{\mathrm{k}}$ ), donde $d_{k}$ es un hiperparámetro que tomó valores $d_{1}=1$ y $d_{2}=5$. También se usó $d_{2}=0,1$ obteniendo iguales resultados.

- Para el efecto aleatorio que captura la estructura espacial $\left(b_{i}\right)$ se asumió una distribución espacial normal con estructura CAR como se especificó en (3).

- Para el efecto aleatorio que captura la estructura temporal $\left(W_{i j}\right)$ se asumió una distribución NM con la estructura de covarianza especificada en (6).

Las distribuciones condicionales a posteriori fueron estimadas por medio del algoritmo Monte Carlo en Cadenas de Márkov ${ }^{32,33}$. Los estadísticos resumen [media, desviación estándar (DE), mediana e intervalos de credibilidad del 95\%, IC $95 \%{ }_{\text {Cred }}$ ] de la distribución a posteriori conjunta se obtuvieron simulando grandes muestras. Fueron generadas 1.100.000 muestras de cada parámetro de interés, siendo descartadas las primeras 100.000 para evitar el efecto de los valores iniciales (burn-in samples). Posteriormente, para la obtención de los estimadores de Monte Carlo de las medias a posteriori de interés, se generaron 1.000.000 de muestras y se escogieron 10.000 (con saltos de 100 para evitar correlación entre las muestras generadas sucesivamente).

La convergencia de los algoritmos de simulación se verificó a partir de la inspección de gráficos (traceplots).

\section{Resultados}

En este estudio se analizaron 140.734 defunciones por ECV ocurridas en los municipios de la región Pacifico de Colombia durante el periodo 20022015 (70.987 en hombres y 69.747 en mujeres) que representaron el $24,4 \%$ y $33,4 \%$ del total de muertes masculinas y femeninas, respectivamente.

La tasa de mortalidad estandarizada por ECV (x100.000) durante el periodo 2002-2015 fue 120,0 en hombres y 112,2 en mujeres. Aunque en ambos grupos las tasas crudas de mortalidad por ECV aumentaron durante el periodo 2002-2015 (hombres de 128,1 a 146,0; mujeres de 127,4 a 143,2), las tasas estandarizadas mostraron una tendencia descendente al pasar de 129,0 a 119,3 en hombres (Figura 2a) y de 129,0 a 110,0 en mujeres (Figura 2b). Por departamentos (Figura 2a y Figura 2b) las mayores tasas de mortalidad se observaron en el Valle del Cauca, donde también hubo una tendencia descendente al igual que en Cauca. Por el contrario en Nariño y Chocó se observaron fluctuaciones con tendencias casi estables.

En ambos sexos la principal causa de muerte fueron las enfermedades isquémicas siendo mayor en hombres (Figura 2c) que en mujeres (Figura $2 \mathrm{~d}$ ) (tasa anual promedio de 60,4 y 46,5, respectivamente). La segunda causa de muertes por ECV fue debida a enfermedades cerebrovasculares (hombres: 28,2; y mujeres: 32,6) y la tercera fueron las enfermedades hipertensivas (hombres: 13,4; y mujeres: 17,0).

La tasa de mortalidad se mantuvo por debajo de 10 hasta los 30 años en hombres y 35 en mujeres, y a partir de los 50 años en hombres y 55 en mujeres superaron el valor de 100 muertes $\mathrm{x}$ 100.000 habitantes. La distribución espacial de la REM para el periodo de estudio mostró mayor riesgo de mortalidad por ECV en los municipios del centro de la región (Valle del Cauca) tanto en hombres (Figura 3a) como en mujeres (Figura 3b), además en la capital de Chocó (Quibdó) principalmente en mujeres.

La distribución espacial de los indicadores socioeconómicos en los 177 municipios de la región Pacifico (Figura 4) mostró predominio de municipios pequeños en más del $90 \%$ de la región (Figura 4a). Los municipios más pobres de acuerdo con los indicadores NBI (Figura 4b), IPM (Figura 4d) y DEVI (Figura 4e) se encuentran en el norte de la región (departamento de Chocó) y en el sur (departamentos de Cauca y Nariño). En general, la región central (Valle del Cauca) mostró las mejores condiciones socioe- 
a) Hombres

180

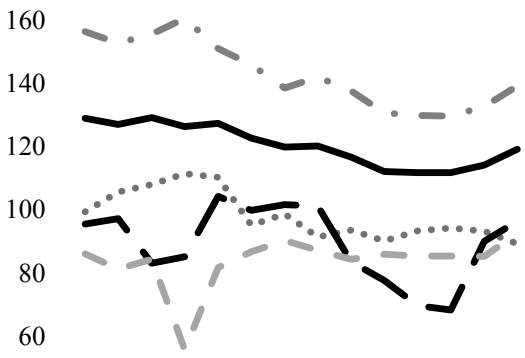

40

20

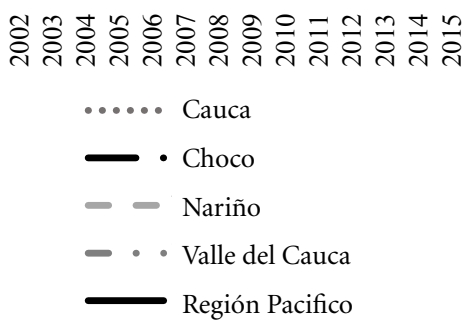

c) Hombres

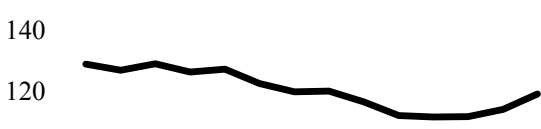

100

80

60

40

20

0

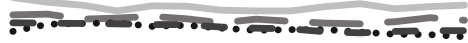

융

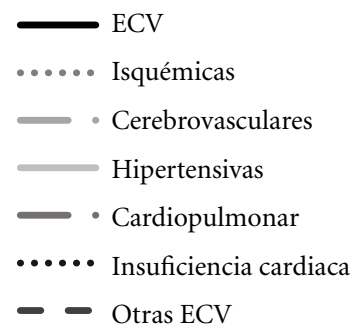

b) Mujeres

180

160

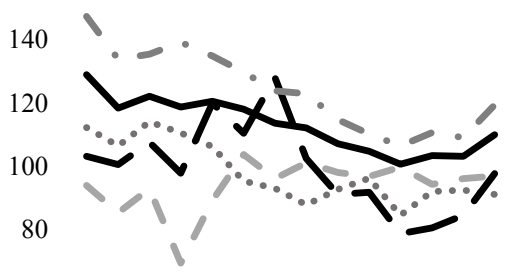

60

40

20

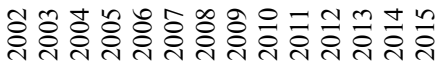

...... Cauca

- Choco

- - Nariño

- - Valle del Cauca

Región Pacifico

d) Mujeres

140

120

100

80

60

40

20

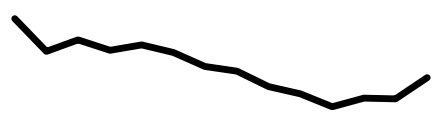
(1)

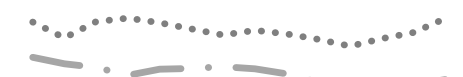$$
\text { .. }
$$

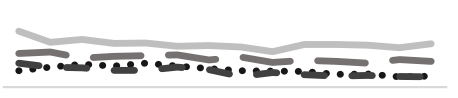

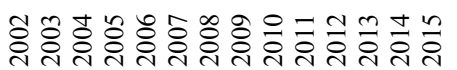

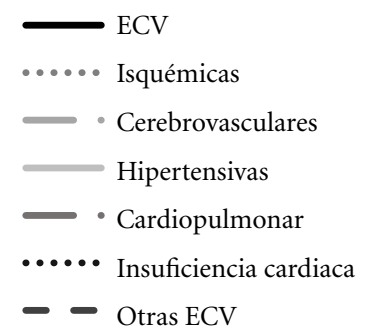

Figura 2. Tasas estandarizadas de mortalidad (x100.000), según sexo, por ECV en la región Pacifico de Colombia. ECV por departamentos: a) hombres y b) mujeres. ECV y principales causas por sexo: $c$ ) hombres y d) mujeres.

Fuente: Desarrollada por los autores. 
a) Hombres

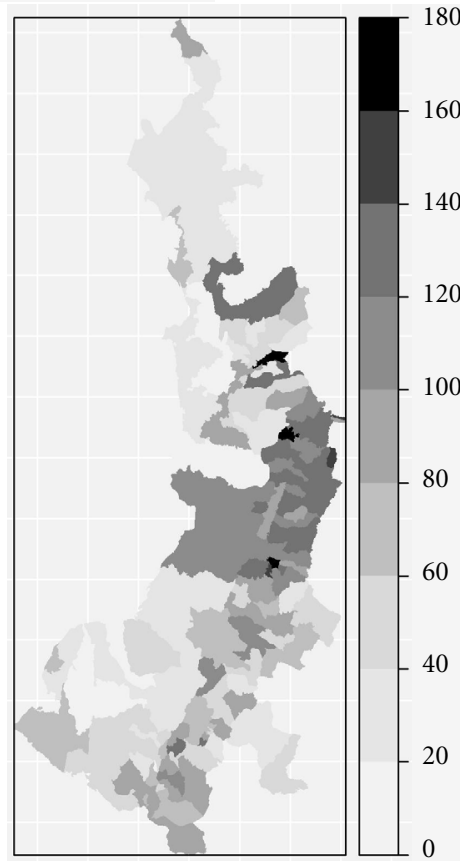

b) Mujeres

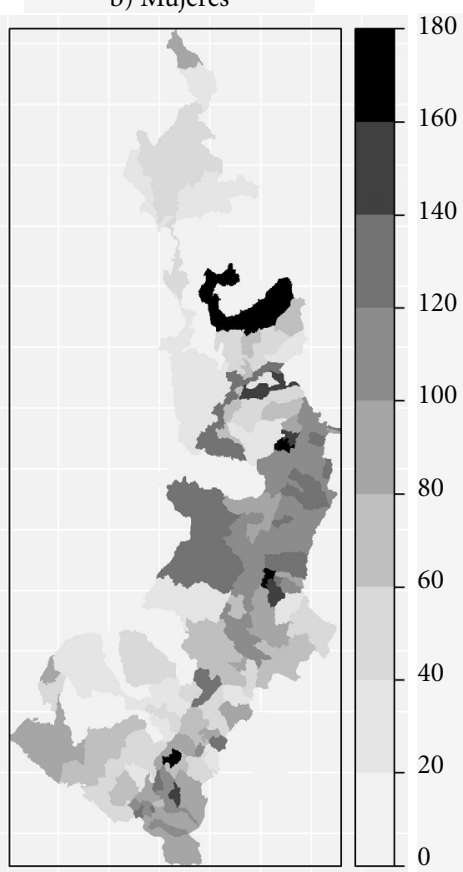

Figura 3. Razón de mortalidad estandarizada (REM), según sexo, en la región Pacifico de Colombia durante el periodo 2002-2015: a) hombres y b) mujeres.

Fuente: Desarrollada por los autores.

a) Categorias
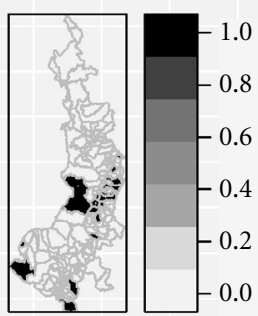

e) DEVI

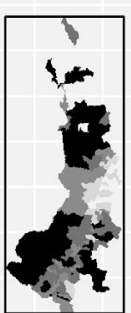

b) IDH

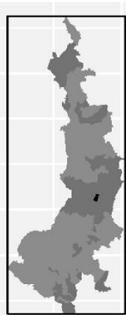

f) Cob.Salud

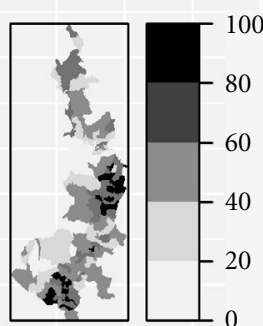

c) NBI

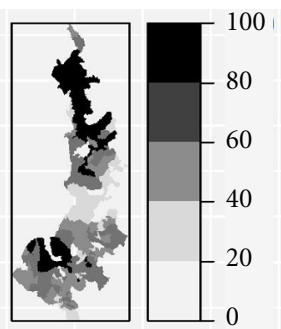

g) Tasa.Alfab

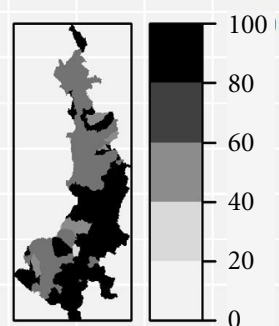

Figura 4. Mapas de las condiciones sociales y económicas de los municipios de la región Pacifico de Colombia: a) Categoría de los municipios; b) Índice de desarrollo humano (IDH); c) Îndice de necesidades básicas insatisfechas (NBI); d) Índice de pobreza multidimensional (IPM); e) Déficit de vivienda (DEVI); f) Cobertura en salud; y g) Tasa de alfabetismo. 
conómicas, así como la mejor cobertura en salud (Figura 4f) y las mayores tasas de alfabetismo (Figura $4 \mathrm{~g})$.

Para determinar la relación entre la mortalidad por ECV y las condiciones socioeconómicas municipales se estimaron los parámetros del modelo espacio-temporal de la ecuación (2). El análisis se centró en el efecto de los indicadores socioeconómicos $\left(X_{1}\right.$ a $\left.X_{7}\right)$ representado por los coeficientes $\beta_{1 j}$ a $\beta_{7 j}$, respectivamente. Bajo el enfoque Bayesiano los parámetros $\left(\beta_{k j}\right)$ se consideran con efecto significativo cuando su IC $95 \%_{\mathrm{Cred}}$ no contiene el cero. Por cuestión de espacio no fue posible mostrar los 98 parámetros estimados en cada uno de los tres modelos.

Los resultados de la modelación jerárquica Bayesiana mostraron algunos indicadores socioeconómicos (categoría del municipio, NBI, IPM y DEVI) significativamente asociados con la mortalidad por ECV. Se encontró una tendencia de mayor mortalidad promedio por ECV en los municipios pequeños (categoría=0) en comparación con los municipios no pequeños (categoría $=1$ ); en los municipios con mayores necesidades básicas insatisfechas (mayor NBI) en comparación con los más favorecidos (menor $\mathrm{NBI}$ ); en los municipios con mayor pobreza multidimensional (mayor IPM) en comparación con los municipios más favorecidos (menor IPM); y también en los municipios con mayor déficit de vivienda (mayor DEVI), en comparación con los que tienen menor déficit (menor DEVI).

Los otros indicadores socioeconómicos (cobertura en salud, IDM y tasa de alfabetismo), aunque no mostraron estimaciones estadísticamente significativas, mostraron tendencias de mayor mortalidad en los municipios menos favorecidos. En promedio, se observó mayor mortalidad por ECV en los municipios con menor cobertura en salud, en comparación con los de mayor cobertura; en los municipios menos desarrollados (menor IDM) en comparación con los más desarrollados (mayor IDM); y en los municipios con menores tasas de alfabetización, en comparación con los de más alfabetización.

\section{Discusión}

En este estudio se analizó espacial y temporalmente la mortalidad por ECV en los municipios de la región Pacifico de Colombia durante el periodo 2002-2015. Además, usando modelos jerárquicos Bayesianos que consideran la posible dependencia espacial y temporal se correlacionaron datos de conteo de defunciones por ECV (ajustados por tamaño poblacional) contra algunos indicadores socioeconómicos municipales (Cuadro 1).

Se encontraron mayores tasas estandarizadas de mortalidad por ECV en los hombres, además de una tendencia descendente en la mortalidad por ECV en ambos sexos, con las mayores tasas ocurriendo en el Valle del Cauca. Las principales causas de muerte por ECV fueron las enfermedades isquémicas del corazón, seguidas de las cerebrovasculares y las hipertensivas. El riesgo de mortalidad por ECV aumentó con la edad, principalmente a partir de los 50 años. La distribución espacial del REM mostró mayor riesgo de ECV en los municipios del Valle del Cauca en ambos sexos, aunque también en las mujeres de Quibdó (Chocó).

Las condiciones socioeconómicas de la región Pacifico colombiana fueron muy heterogéneas, con predominio de municipios pequeños y los más pobres (según el NBI, IPM y DEVI) localizados en los departamentos de Chocó, Cauca y Nariño.

Se encontraron asociaciones significativas entre los niveles de mortalidad por ECV y las condiciones socioeconómicas municipales. En general, el riesgo de mortalidad por ECV fue mayor en los municipios menos favorecidos y con menores niveles de desarrollo económico y social.

Las tasas de mortalidad por ECV (x 100.000) de la región Pacifico colombiana fueron significativamente menores que las tasas de mortalidad por ECV en 2015 a nivel Global 286 (280-291), en América Latina Andina 157 (146-168) 3 y en las Américas en 2016 de 150,7 $7^{34}$, pero mayores que la reportada para Colombia entre 1998 y 2011 con un promedio de 106,2 x 100.0006.

En este estudio las tasas de mortalidad por ECV fueron mayores en hombres que en mujeres como también se ha reportado previamente para las Américas en 2016 (185,2 vs 121,6 $)^{34}$ y en Colombia durante los periodos $1990-2001^{7,35}$ y 1998-20116 . En este estudio las tasas de mortalidad por enfermedades isquémicas fueron mayores en hombres que en mujeres (promedio del periodo 60,4 vs 46,5 ), comportamiento también observado en Colombia durante el periodo 19902001 con tasas de mortalidad en el 2001 de 64,6 en hombres y 42,7 en mujeres ${ }^{35}$.

La tendencia ascendente en las tasas crudas de mortalidad por ECV en hombres y mujeres, pero descendente en las tasas estandarizadas, es explicado por un envejecimiento poblacional ${ }^{6}$. La tendencia descendente en las tasas estandarizadas de mortalidad por ECV observada en hombres y 
mujeres es consistente con la tendencia de América Latina que durante el periodo 2000 a 2009 descendió de 229,9 a 191,4 muertes por 100.000 habitantes ${ }^{34}$. En Colombia también fueron observadas tendencias descendentes en la mortalidad por ECV durante los periodos 1990-2001 de 108,0 a $103,8^{7}$ y también entre 1998-2011 de 108,6 a 95,96. Aunque en el periodo 1990-2001 se observaron tendencias descendentes para las ECV en Colombia, y dentro de este grupo en las enfermedades hipertensivas e insuficiencia cardiaca, contrariamente en este mismo periodo aumentaron las tasas de mortalidad por enfermedades isquémicas del corazón y enfermedades cerebrovasculares ${ }^{35}$.

En este estudio las principales causas de muerte por ECV fueron las enfermedades isquémicas y las cerebrovasculares las cuales también son reconocidas como las principales causas de muerte en el mundo ${ }^{36}$, en América ${ }^{37}$ y en Colombia $^{6,38}$. Durante el periodo 1990-2001 en Colombia las principales ECV fueron las enfermedades isquémicas del corazón, las enfermedades cerebrovasculares, las enfermedades hipertensivas y la insuficiencia cardiaca ${ }^{7,35}$.

El riesgo de mortalidad por ECV aumentó con la edad en hombres y mujeres. Esto puede ser explicado por el envejecimiento poblacional que trae consigo un deterioro del organismo y con ello mayor riesgo de incidencia y mortalidad por ENT incluyendo las ECV. Otros estudios en Colombia también han reportado mayor riesgo de mortalidad por ECV aumentando con la edad, con tasas que empiezan a aumentar a partir del grupo 15-44 años, siguen en ascenso en el grupo 45-64 años y alcanzan el valor más alto en el grupo mayor de 65 años ${ }^{7}$. Este patrón también ha sido observado en enfermedades del grupo de ECV como las isquémicas, cerebrovasculares, hipertensivas e insuficiencia cardiaca donde el riesgo de mortalidad se acelera fuertemente con la edad ${ }^{35}$.

Las mayores tasas de mortalidad observadas en el Valle del Cauca son consistentes con reportes previos en Colombia. Durante el periodo 1998-2011 el Valle del Cauca presentó las mayores tasas de mortalidad por ECV de Colombia

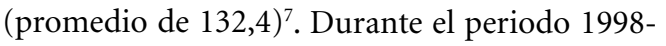
2011 las tasas estandarizadas de mortalidad por ECV variaron de 123,7 a 102,3 en el Valle del Cauca; de 88,9 a 79,9 en Cauca; de 76,6 a 71,0 en Chocó; y de 56,4 a 76,4 en Nariño ${ }^{6}$. Se resalta que durante el periodo 1998-2011 Nariño fue el único departamento de la región que mostró una tendencia ascendente, similar a la tendencia casi estable de nuestro estudio 2002-2015. Además durante el periodo 1991-2001 el Valle del Cauca también presentó las mayores tasas de mortalidad por enfermedades cerebrovasculares de Colombia (promedio de 42,1) ). $^{35}$.

La RME fue mayor en los municipios del Valle del Cauca y también en las mujeres de Quibdó (capital del Chocó). El riesgo aumentado de mortalidad por ECV en el Valle del Cauca coincide con su nivel de desarrollo, sabiendo que es el tercer departamento más industrializado del país y donde la literatura muestra mayor incidencia y mortalidad de ENT en las regiones más desarrolladas asociadas a procesos de transición demográfica y epidemiológica, urbanización e industrialización ${ }^{7,35}$. Las altas tasas de mortalidad por ECV en las mujeres de Chocó estuvieron relacionadas a las enfermedades cerebrovasculares e hipertensivas, donde estas últimas son más prevalentes en poblaciones afrodescendientes que caracteriza al departamento de Chocó ${ }^{35}$.

La asociación entre mayores niveles de mortalidad por ECV y peores condiciones sociales y económicas de los municipios coinciden con diversas investigaciones realizadas por la OPS y por otros autores donde sistemáticamente se observan los peores indicadores de salud en los grupos y clases más desfavorecidas ${ }^{39,40}$.

Son diversas las investigaciones sobre el tema de las desigualdades sociales en salud donde sus hallazgos muestran que la mortalidad general y diversas causas específicas de muerte suelen concentrarse en las regiones más pobres y marginadas. El fenómeno que los pobres presentan peor estado de salud es mundial y con evidencia probatoria proveniente de países de ingresos bajos, medianos y altos ${ }^{41}$.

En Colombia, las regiones con mayores niveles de pobreza y privación socioeconómica también tienen mayor riesgo de muerte reflejado en mayores tasas de mortalidad por diversas causas 9 . Un estudio que analizó la mortalidad por causas externas en los municipios de Antioquia durante el periodo 2000-2010 y su asociación con las condiciones socioeconómicas de los municipios, usando los indicadores NBI e IDH y modelos jerárquicos Bayesianos, encontró un gradiente social negativo tanto en hombres como en mujeres, además de mayor riesgo de muerte por causas externas en los municipios más pobres (mayor NBI) y menos desarrollados (menor IDH) ${ }^{9}$.

En Colombia también se encuentra evidencia de estudios descriptivos que soporta la relación entre el estado de salud y las condiciones socioeconómicas municipales. Un estudio en el 
Valle del Cauca identificó desigualdades en la mortalidad por VIH y cáncer (próstata y útero) según dos indicadores socioeconómicos (NBI y valor agregado municipal) encontrando mayores tasas de mortalidad en los municipios con mayor índice de $\mathrm{NBI}^{13}$. Otro estudio realizado en el departamento del Huila para determinar desigualdades en la mortalidad por causas especificas relacionadas con características socioeconómicas de sus municipios durante el periodo 2009-2013 encontró asociación entre mortalidad (cáncer gástrico, por enfermedades hipertensivas, homicidios y en la mortalidad en niñez) y las variables socioeconómicas evaluadas, con mayores tasas de mortalidad en los municipios menos favorecidos (con más baja cobertura en educación media y con mayores NBI) ${ }^{21}$.

Específicamente sobre las desigualdades sociales en la mortalidad por ECV se encontró un estudio en municipios del eje cafetero de Colombia (departamentos de Caldas, Quindío y Risaralda) que también comparó las desigualdades en la mortalidad por enfermedades cardiovasculares (hipertensivas, isquémicas y cerebrovasculares) durante el periodo 2009-2011 con indicadores socioeconómicos de los municipios (NBI e PIB municipal per cápita) encontrando mayor riesgo de morir por enfermedad isquémica e hipertensiva en los municipios más pobres (mayor NBI) ${ }^{21}$.

Además de los factores de riesgo comportamentales que aumentan el riesgo de incidencia y mortalidad por ECV como el consumo alcohol y tabaco, y la obesidad principalmente como resultado de una mala alimentación y el sedentarismo $^{2,7}$, existen otros determinantes de las ECV asociados con el contexto geográfico de las personas.

Las desigualdades socioeconómicas son factores determinantes de la exposición al riesgo de ECV tanto en el nivel individual (factores comportamentales) como en el colectivo (factores estructurales). Los efectos de los factores comportamentales cada vez más recaen en las regiones y poblaciones más pobres donde es más difícil adoptar estilos de vida saludables. La pobreza y las ECV crean un círculo vicioso donde los pobres se exponen más a los factores de riesgo como tabaquismo, alcohol, dieta malsana y sedentarismo que conducen a las ECV y agravan la pobreza ${ }^{2}$.

Los municipios menos favorecidos social y económicamente pueden enfrentar sistemas de salud más débiles con barreras de acceso a servicios de salud eficientes, oportunos y equitativos, falta de acceso a programas preventivos y tratamiento oportuno, entre otros ${ }^{38}$. Las regiones pe- riféricas de Colombia que incluye a municipios de Chocó, Cauca, Nariño y algunos del Valle del Cauca se caracterizan, entre otros, por presentar mayor concentración de pobreza, menores valores de PIB per cápita, menores recursos propios, mayores necesidades básicas insatisfechas, menor coberturas de servicios públicos básicos, mayores tasas de analfabetismo, mayor desplazamiento forzado, menor participación política y mayor corrupción, así como peores resultados en indicadores de salud como la tasa de mortalidad infantil y la esperanza de vida al nacer ${ }^{14,26}$.

La principal limitación de este estudio correspondió al uso de fuentes secundarias de información en lo referente a defunciones por ECV, tamaños poblacionales e indicadores socioeconómicos. Los datos de mortalidad provienen de las Estadísticas Vitales del DANE que, a pesar de ser oficiales y consolidados, presentan problemas de calidad y cobertura ${ }^{42}$. Estimaciones de la calidad de la certificación para las muertes cardiovasculares mal definidas fueron muy bajas durante el periodo 2007-2011, siendo de 1,8\% en Cauca y Chocó, 2,4\% en Nariño y 1,6\% en Valle del Cauca $^{42}$. Además se espera que las defunciones presenten menor calidad en los municipios más pequeños donde los certificados de defunción no siempre son diligenciados por médicos 9 . Las estimaciones de cobertura de mortalidad en el 2002 fueron de 64,5\% en Chocó, 80,7\% en Nariño, $82,3 \%$ en Cauca y 93,7\% en Valle del Cau$\mathrm{ca}^{43}$. Los tamaños poblacionales para calcular las tasas de mortalidad son proyecciones oficiales del DANE a partir del último censo en Colombia en 2005.

Otra limitación de este estudio estuvo relaciona con la medición transversal de las condiciones socioeconómicas para los 177 municipios con información del 2005, año en que fueron calculados la mayoría de los indicadores usando datos del último censo en Colombia. Con esto, se asumió que las condiciones socioeconómicas de los municipios permanecieron contantes durante el periodo 2002-2015, lo cual seguramente no refleja de la realidad.

La principal fortaleza de este estudio fue la integración de herramientas de sistemas de información geográfica (SIG), modelamiento estadístico Bayesiano, información de mortalidad e indicadores socioeconómicos para el estudio de las desigualdades geográficas en la mortalidad por ECV en la región Pacifico de Colombia.

En este estudio se utilizaron técnicas de regresión espacio-temporal mediante un modelo Poisson con estructura CAR que consideró la 
dependencia espacial en los datos además de un Proceso Gaussiano para capturar la tendencia temporal de los 14 años $^{44}$. A nuestro conocimiento este es el primer estudio sobre desigualdades sociales en la mortalidad por ECV en la región Pacifico, y su relación con indicadores socioeconómicos municipales, que usa modelos jerárquicos Bayesianos para el estudio espacial y temporal de la mortalidad durante un periodo de 14 años.

\section{Colaboradores}

Todos los autores participaron en el estudio y aprobaron la versión final del manuscrito. M Pérez-Flórez participó en la concepción y diseño del estudio; análisis e interpretación de los resultados; redacción del artículo; y aprobación de la versión final enviada para publicación. JA Achcar participó en la concepción y diseño del estudio; análisis e interpretación de los resultados; revisión crítica del artículo; y aprobación de la versión final enviada para publicación. 


\section{Referencias}

1. World Health Organization (WHO). Noncommunicable Diseases Country Profiles 2018. Ginebra: WHO; 2018.

2. Organizacion Mundial de la Salud (OMS). Informe sobre la situación mundial de las enfermedades no transmisibles 2010. Resumen de orientación. Ginebra: OMS; 2011.

3. Roth GA, Johnson C, Abajobir A, Abd-Allah F, Abera SF, Abyu G, Ahmed M, Aksut B, Alam T, Alam K, Alla F, Alvis-Guzman N, Amrock S, Ansari H, Ärnlöv J, Asayesh H, Atey TM, Avila-Burgos L, Awasthi A, Banerjee A, Barac A, Bärnighausen T, Barregard L, Bedi N, Ketema EB, Bennett D, Berhe G, Bhutta Z, Bitew S, Carapetis J, Carrero JJ, Malta DC, Castañeda-Orjuela CA, Castillo-Rivas J, Catalá-López F, Choi J-Y, Christensen H, Cirillo M, Cooper Jr L, Criqui M, Cundiff D, Damasceno A, Dandona L, Dandona R, Davletov K, Dharmaratne S, Dorairaj P, Dubey M, Ehrenkranz R, El Sayed Zaki M, Faraon EJA, Esteghamati A, Farid T, Farvid M, Feigin V, Ding EL, Fowkes G, Gebrehiwot T, Gillum R, Gold A, Gona P, Gupta R, Habtewold TD, Hafezi-Nejad N, Hailu T, Hailu GB, Hankey G, Hassen HY, Abate KH, Havmoeller R, Hay SI, Horino M, Hotez PJ, Jacobsen K, James S, Javanbakht M, Jeemon P, John D, Jonas J, Kalkonde Y, Karimkhani C, Kasaeian A, Khader Y, Khan A, Khang Y-H, Khera S, Khoja AT, Khubchandani J, Kim D, Kolte D, Kosen S, Krohn KJ, Kumar GA, Kwan GF, Lal DK, Larsson A, Linn S, Lopez A, Lotufo PA, El Razek HMA, Malekzadeh R, Mazidi M, Meier T, Meles KG, Mensah G, Meretoja A, Mezgebe H, Miller T, Mirrakhimov E, Mohammed S, Moran AE, Musa KI, Narula J, Neal B, Ngalesoni F, Nguyen G, Obermeyer CM, Owolabi M, Patton G, Pedro J, Qato D, Qorbani M, Rahimi K, Rai RK, Rawaf S, Ribeiro A, Safiri S, Salomon JA, Santos I, Milicevic MS, Sartorius B, Schutte A, Sepanlou S, Ali Shaikh M, Shin M-J, Shishehbor M, Shore H, Silva DAS, Sobngwi E, Stranges S, Swaminathan S, TabarésSeisdedos R, Atnafu NT, Tesfay F, Thakur JS, Thrift A, Topor-Madry R, Truelsen T, Tyrovolas S, Ukwaja KN, Uthman O, Vasankari T, Vlassov V, Vollset SE, Wakayo T, Watkins D, Weintraub R, Werdecker A, Westerman R, Wiysonge CS, Wolfe C, Workicho A, Xu G, Yano Y, Yip P, Yonemoto N, Younis M, Yu C, Vos T, Naghavi M, Murray C. Global, Regional, and National Burden of Cardiovascular Diseases for 10 Causes, 1990 to 2015. J Am Coll Cardiol 2017; 70(1):1-25.

4. Frenk J, Frejka T, Bobadilla JL, Lozano R, Sepúlveda J. La transición epidemiológica en América Latina. Boletín la Of Sanit Panam 1991; 6(111):485-496.

5. Mora-Pabón G. Research on heart failure in Colombia, time to take a step forward. Rev Fac Med 2018; 66(2):137-138.

6. Boletín ONS No.1 Diciembre 2013: Enfermedad cardiovascular: principal causa de muerte en Colombia [Internet]. 2013 [cited 2019 may 10]. Available from: www.ins.gov.co.

7. González M. Mortalidad por enfermedades crónicas no transmisibles en Colombia de 1990 a 2001. Inf Quinc Epidemiol Nac 2007; 12:177-187.
8. Whitehead M. The concepts and principles of equity in health. Int J Heal Serv 1992; 22(2):429-445.

9. Caicedo-Velásquez B, Álvarez-Castaño LS, MaríDell'Olmo M, Borrell C. Evolución de las inequidades en mortalidad por causas externas entre los municipios de Antioquia (Colombia). Gac Sanit 2016; 30(4):279-286.

10. Gomez Arias RD. La mortalidad evitable como indicador de desempeño de la política sanitaria Colombia. 1985-2001. Medellin: Imprenta Universidad de Antioquia; 2008.

11. Baena A, Almonte M, Valencia ML, Martinez S, Quintero K, Sanchez GI. Tendencias e indicadores sociales de la mortalidad por cáncer de mama y cuello uterino. Antioquia, Colombia, 2000-2007. Salud Publica Mex 2011; 53(6):486-492.

12. Fernández Ortiz YN. Desigualdades en la morbilidad (2002-2006) y mortalidad (2006-2010) por cáncer de mama en Colombia. Bogotá: Pontificia Universidad Javeriana; 2015.

13. Cuesta F, Gonzales P, Nieto E, Alzate G. Desigualdades sociales en la mortalidad por VIH y tumores malignos en municipios del departamento de Valle del Cauca (Colombia), según indicadores económicos 20092013. Arch Med 2018; 18(2):373-384.

14. Cortés D, Vargas JF. Inequidad Regional en Colombia. Documentos CEDE 34. CEDE Centro de Estudios sobre Desarrollo Económico. Colombia: Ediciones Uniandes; 2012.

15. Martínez J, San Sebastián M, Labonte R, Mosquera PA, Hernández J, Sanders D, San Sebastián M. The impact of primary healthcare in reducing inequalities in child health outcomes, Bogotá - Colombia: an ecological analysis. Int J Equity Health 2012; 11(1):66.

16. Jaramillo-Mejía MC, Chernichovsky D, Jiménez-Moleón JJ. Brechas regionales de la mortalidad infantil en Colombia. Rev Peru Med Exp Salud Publica 2018; 30(4):6-11.

17. Sandoval-Vargas YG, Eslava-Schmalbach JH. Inequidades en mortalidad materna por departamentos en Colombia para años 2000-2001, 2005-2006 y 20082009. Rev Salud Publica 2013; 15(4):529-541.

18. Cárdenas-Cárdenas LM, Cotes-Cantillo K, ChaparroNarváez PE, Fernández-Niño JA, Paternina-Caicedo A, Castañeda-Orjuela C, De la Hoz-Restrepo F. Maternal Mortality in Colombia in 2011: A Two Level Ecological Study. PLoS One 2015; 10(3):e0118944.

19. Cardona D, del Pilar Cerezo M, Parra H, Quintero L, Muñoz L, Cifuentes OL, Vélez SC. Desigualdades en la mortalidad por enfermedades cardiovasculares en los municipios del Eje Cafetero, 2009-2011. Biomédica 2015; 35(3):379-394.

20. Rodríguez García J. Desigualdades socioeconómicas entre departamentos y su asociación con indicadores de mortalidad en Colombia en 2000. Rev Panam Salud Pública 2007; 21(2-3):111-124.

21. Cardona-Rivas D, Montalvo-Arce CA, Carmona-Patiño CA. Desigualdades en mortalidad relacionadas con características socioeconómicas en el departamento del Huila, Colombia 2009-2013. Rev Fac Nac Salud Publica 2018; 35(3):343-357. 
22. Dirección de Epidemiología y Demografía. Grupo ASIS. Análisis de Situación de Salud según regiones Colombia. Colombia: Ministerio de Salud y Protección Social: 2013.

23. Programa de las Naciones Unidas para el Desarrollo (PNUD). Objetivos de Desarrollo del Milenio. Informe 2015. Nueva York: PNUD; 2015.

24. Baptista EA, Queiroz BL. Spatial Analysis of Mortality by Cardiovascular Disease in the Adult Population: A Study for Brazilian Micro-Regions Between 1996 and 2015. Spat Demogr 2019; 7(1):83-101.

25. Departamento Administrativo Nacional de Estadística (DANE) Colombia. Proyecciones de población municipales por área 2005-2020 [Internet]. 2011 [cited 2017 Sep 11]. Available from: https://goo.gl/5Ghk8n.

26. Galvis LA, Roca AM. Persistencia de las desigualdades regionales en Colombia: Un análisis espacial. Documentos de Trabajo Sobre Economía Regional; 2010.

27. Dirección de Censos y Demografía. Estadísticas Vitales [Internet]. Bogotá; 2009 [cited 2017 Sep 11]. Available from: https://www.dane.gov.co/files/investigaciones/ fichas/Estadisticas_vitales.pdf.

28. Pan American Health Organization (PAHO). New PAHO List 6/67 for Tabulation of ICD-10 Mortality Data. Epidemiol Bull 1999; 20(3):4-9.

29. Antònia Barceló M, Saez M, Cano-Serral G, Ángel Martínez-Beneito M, Miguel Martínez J, Borrell C, Ocaña-Riola R, Montoya I, Calvo M, López-Abente G, Rodríguez-Sanz M, Toro S, Alcalá JT, Saurina C, Sánchez-Villegas P, Figueiras A. Métodos para la suavización de indicadores de mortalidad: aplicación al análisis de desigualdades en mortalidad en ciudades del Estado español (Proyecto MEDEA). Gac Sanit 2008; 22(6):596-608.

30. Achcar JA, Martinez EZ, Souza ADP, Tachibana VM, Flores EF. Use of Poisson spatiotemporal regression models for the Brazilian Amazon Forest: malaria count data. Rev Soc Bras Med Trop 2011; 44(6):749-754.

31. Spiegelhalter D, Thomas A, Best N, Lunn D. WinBUGS User Manual. Cambridge: MRC Biostatistics Unit.; 2003.

32. Gelfand AE, Smith AFM. Sampling-based approaches to calculating marginal densities. J Am Stat Assoc 1990; 85(410):398-409.

33. Chib S, Greenberg E. Understanding the Metropolis -Hastings Algorithm. Am Stat 1995; 49(4):327-335.

34. Fernando L, Pamela S, Alejandra L. Cardiovascular Disease in Latin America: The Growing Epidemic. Prog Cardiovasc Dis 2014; 57(3):262-267.

35. González M. Mortalidad por enfermedades cardiovasculares en Colombia de 1990 a 2001. In: Inf Quinc Epidemiol Nac [Internet]. Bogotá; 2007 [cited 2017 Sep 11]. p. 145-187. Available from: https://www.ins. gov.co.

36. Organización Mundial de la Salud (OMS). Las 10 principales causas de defunción [Internet]. [cited 2017 Sep 11]. Available from: http://www.who.int/mediacentre/factsheets/fs310/es/.
37. Pan American Health Organization (PAHO). World Health Organization (WHO). Noncommunicable diseases in the Region of the Americas: facts and figures. Washington, D.C., Ginebra; 2019.

38. Martínez JC. Factores asociados a la mortalidad por enfermedades no transmisibles en Colombia, 20082012. Biomédica 2016; 36:535-546.

39. Kawachi I, Subramanian S, Almeida-Filho N. A glossary for health inequalities. J Epidemiol Community Heal 2002; 56:647-652.

40. Rojas-Esguerra DF, Zarama-Tobar IL, Borrero-Ramírez YE, Arrivillaga-Quintero M. Revisión de la producción académica latinoamericana sobre desigualdades en mortalidad 2007-2014. Rev Gerenc Polit Salud 2017; 16(32):10-32.

41. Organización Panamericana de la Salud (OPAS). Determinantes e inequidades en salud. In: Salud en las Americas, Edicion de 2012: Volumen regional. Washington, D.C.: OPAS; 2012. p. 12-59.

42. Cendales R, Pardo C. Calidad del certificado de defunción en Colombia. Colomb Med 2018; 49(9):121-127.

43. Departamento Administrativo Nacional de Estadística (DANE). Dirección de Censos y Demografía (DCD). La Mortalidad Materna y Perinatal en Colombia en los albores del siglo XXI [Internet]. 2006 [cited 2017 Sep 11]. Available from: http://docplayer. es/40107048-Estudio-la-mortalidad-materna-y-perinatal-en-colombia-en-los-albores-del-siglo-xxi.html.

44. Lawson AB. Bayesian disease mapping: Hierarchical modeling in spatial epidemiology. Bayesian Disease Mapping: Hierarchical Modeling in Spatial Epidemio$\log y$. Charleston: Chapman and Hall/CRC; 2008.

Artículo presentado en 22/11/2019

Aprobado en 18/03/2020

Versión final presentada en 20/03/2020

Editores jefes: Romeu Gomes, Antônio Augusto Moura da Silva 